\title{
L'occupation et l'aménagement des espaces à risque en milieu urbain équatorial : le cas des fonds de vallées à Yaoundé (Cameroun)
}

\author{
H. Blaise Nguendo Yongsi, Université de Montréal \\ Antoine Lutumba Ntetu, Université du Québec à Chicoutimi
}

\section{Introduction}

$\Gamma^{9}$ urbanisation constitue un événement démographique, géographique, social, voire politique majeur que la planète connaît depuis la fin du siècle dernier. Pour bien prendre la mesure du phénomène, rappelons qu'en 1950, moins d'un tiers seulement de la population mondiale était urbaine, soit 740 millions de citadins pour environ 1,8 milliard de ruraux ${ }^{1}$. En 2000, cette population urbaine était de l'ordre de $47 \%$. Et on estime couramment que d'ici 2020, trois habitants de la planète sur quatre vivront en milieu urbain, sous l'hypothèse que chaque jour près de 180000 personnes viennent accroitre la popula-

tion urbaine ${ }^{2}$. Plus encore que ces chiffres globaux, ce qui frappe c'est le processus d'urbanisation extraordinairement rapide des pays en développement, notamment l'Afrique. De fait, l'urbanisation de l'Afrique est, après l'accroissement démographique, le changement le plus spectaculaire qu'ait connu le continent au cours de ces dernières décennies. Aux yeux de certains, c'est aussi le changement le plus préoccupant car, de rural à plus de $80 \%$ en 1960 , le continent est aujourd'hui urbanisé à $41,3 \%$ et l'on estime qu'en 2030 , il affichera un taux de $52,9 \%^{3}$. La rapidité et l'ampleur du phénomène lui ont valu les qualificatifs les plus divers : urbanisation galopante, effrénée, déferlante, sauvage. Bien que longuement débattu, ce phénomène se rapporte toujours à une actualité brûlante dans la mesure où la croissance urbaine et les conséquences écologiques et socio sanitaires qui en découlent constituent des éléments clés de la difficile et dramatique problématique de cette partie de la planète. Si l'explosion urbaine en Afrique paraît préoccupante, c'est sans doute en raison de son caractère non planifié. En effet, la poussée démographique générale des villes africaines, liée aux effets conjugués de l'exode rural et de l'excédent naturel, s'est traduite par l'élévation des densités humaines dans les quartiers d'accueil et par une extension démesurée des vil$\operatorname{les}^{4}$. Parallèlement à cet étalement spatial, on voit surgir aussi des aménagements dans des terrains vacants intra-muros, notamment sur des espaces impropres à la construction du fait des risques écologiques et/ou sanitaires qu'ils présentent. La littérature géographique rapporte des cas d'aménagement réalisé sur lesdits sites. Ainsi en est-il des «Barrancos » de Lima (maisons construites près des dépotoirs), des «favellas » de Rio de Janeiro (constructions édifiées sur les versants abrupts de collines), des bidonvilles de Fès, Caracas ou d'Ahmedabad (habitat construit dans des carrières) ${ }^{5}$. Dans la métropole camerounaise de Yaoundé, ce genre d'aménagement s'observe avec emphase dans les différents fonds de vallées qui parsèment la ville. Le présent article vise principalement à saisir la logique d'occupation d'un tel espace et à présenter les différents types d'aménagement qui y sont réalisés.

\section{Les notions d'espaces à risque et de fonds de vallées}

\section{Le risque}

La notion de risque est polysémique. Elle est généralement définie en fonction de l'objectif de l'étude qu'on veut mener. Ainsi, elle est tantôt reliée à un événement non souhaité, tantôt à une éventualité, tantôt encore à la probabilité de se tromper. Mais dans son acception écologique - celle qui nous concerne -, le risque est l'exposition plus ou moins volontaire à 
une situation qui a une probabilité de se produire et dont l'occurrence entraînerait des dégâts ${ }^{6}$. Comprise de la sorte, la notion de risque recouvre deux éléments : l'aléa et l'enjeu. Par aléa, il faut entendre la manifestation d'un phénomène naturel : crues, glissements de terrain, séismes, éruptions volcaniques pour ne citer que ceux propres aux zones intertropicales. Un aléa se caractérise par sa probabilité d'occurrence et par l'intensité de sa manifestation (hauteur et vitesse de l'eau pour les crues, magnitude pour les séismes, largeur de bande pour les glissements de terrain, etc.).

L'enjeu ici est relatif aux dommages directs comptabilisables dès la fin de l'événement exceptionnel sur les habitations, les infrastructures, les cultures ou le cheptel et, dans les cas les plus dramatiques, les vies humaines. Ainsi, un espace à risque renvoie à cette portion du globe terrestre susceptible d'être soumise à un phénomène naturel aux impacts matériel et humain concrets.

\section{Les fonds de vallées}

Morphologiquement, les fonds de vallées sont des terrains bas, des espaces localement déprimés ${ }^{7}$. Dans le cadre régional de Yaoundé, ils constituent des éléments topographiques d'une surface d'aplanissement modelée par l'érosion. À Yaoundé plus exactement, ils correspondent à des dépressions allongées, généralement parcourues par des cours d'eau. C'est pourquoi, dans le cadre de cet article, nous parlerons communément et invariablement de bas-fonds marécageux ou de vallées marécageuses.

\section{La présence des vallées marécageuses dans l'espace urbain de Yaoundé}

Sur le plan géomorphologique, Yaoundé appartient à la zone méridionale du Cameroun. Définie comme une pénéplaine ${ }^{8}$, cette zone est une surface mollement ondulée d'une altitude moyenne de $700 \mathrm{~m}$, appartenant à la grande « Surface africaine I» datée de l'éocène. L'observation de la carte au 1:500 000 montre que la monotonie de ce relief "pénéplané » est rompue par la présence de massifs qui jalonnent la ligne de partage des eaux et même, au nord, par de petites chaînes montagneuses correspondant à des lignes structurales de direction sud-sud-ouest - nord-nord- est. Dans le secteur occupé par les vallées de la Sanaga et du Nyong, une large dorsale entaille la vieille surface "pénéplanée » : c'est la région de Yaoundé. Celle-ci correspond à la dorsale Nyong-Sanaga, morceau de vieux socle au centre duquel est située la ville de Yaoundé.

Dans le détail, les cartes topographiques au 1:50 000 et au 1:10 000 montrent l'aspect morphologique relativement varié du site de Yaoundé. Cette disposition du relief tient des différents phénomènes tectoniques qu'a connus la région. En effet, au Tertiaire, la région de Yaoundé a été le théâtre de rejeux tectométamorphiques qui ont soulevé et basculé le massif vers l'ouest, fracturant et broyant certains secteurs ainsi rapidement exposés à l'altération et à l'érosion'. Ces rejeux ont ainsi mis en place des espaces surélevés appelés ici plateaux, et des espaces déprimés drainés par des cours d'eau appelés fonds de vallées. Le Mfoundi et ses affluents, en s'élargissant vers l'aval, n'ont laissé à leur confluent qu'une place minime aux interfluves qu'elles délimitent. Cette forme d'érosion régressive et latérale a joué sur les dimensions des plateaux et sur les formes des vallées (figure 1).

\section{Logique d'occupation des fonds de vallée à Yaoundé et risques encourus}

S'il est indiscutable que l'Afrique a connu une civilisation urbaine anté-coloniale, c'est néanmoins la colonisation qui lui a imprimé les caractères qu'elle connaît encore aujourd'hui. En effet, la colonisation européenne s'est accompagnée d'une intense création urbaine aussi bien pour des besoins de l'administration des territoires que pour l'exploitation des ressources locales. Le modèle d'urbanisme colonial, sous prétexte d'hygiénisme, va cependant accentuer le caractère ségrégatif de l'habitat et des quartiers des villes africaines en imposant sa trame qui persiste aujourd'hui dans la plupart des agglomérations ${ }^{10}$. Yaoundé connaîtra certes ce «choc colonial qui a constitué un élément décisif [de son] urbanisme ${ }^{11}$ mais, du fait de son site collinaire, le processus d'occupation du sol y a été singulier. À la différence d'autres villes du pays, voire des pays voisins où l'urbanisation a progressé par étalement du noyau central vers les périphéries, l'occupation du site de Yaoundé s'est faite à partir de plusieurs noyaux, et notamment d'anciens villages existants sur les sommets d'interfluves. 


\section{Figure 1 - Vallées et plateaux à Yaoundé}

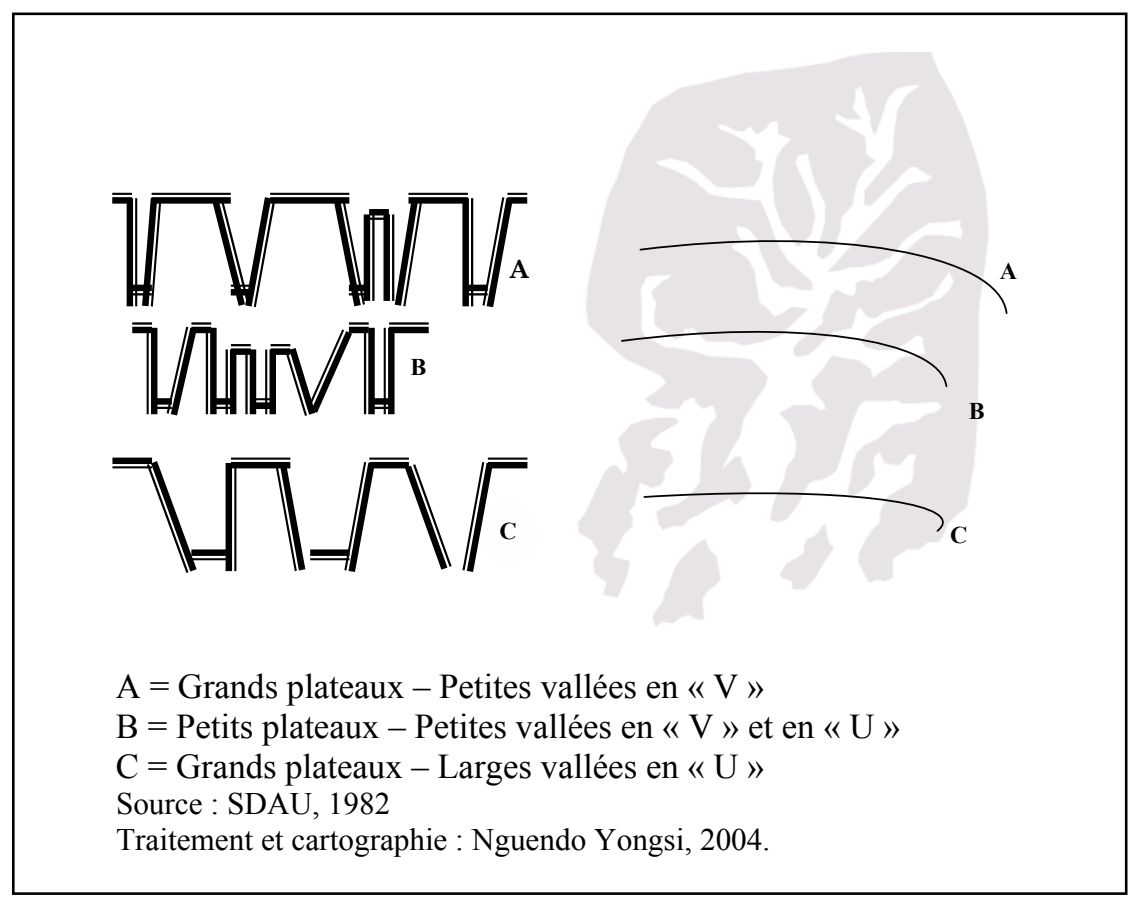

Comme le montre la figure 2, ce processus s'est déroulé en trois étapes : 1) l'étape de l'occupation des sommets de collines (crêtes), 2) l'étape de débordement des crêtes et progression sur les versants et 3 ) l'étape de l'occupation des bas-fonds.

Première étape : l'occupation des sommets de collines (crêtes). C'est le point de départ de l'urbanisation sur le site urbain. Les populations s'installent d'abord sur les lignes de crêtes, bien ventilées, bien drainées et d'accès facile. Cette installation est le fait des autochtones d'abord, des colonisateurs allemands et français ensuite, et enfin des couches sociales favorisées et de quelques étrangers. Ici, l'urbanisation s'appuie sur les voies de pénétration et les villages existants et s'étend en tache d'huile de part et d'autre de ces pistes. Au cours de cette première phase, l'occupation du site laisse encore de très larges espaces libres dédiés aux activités agricoles.

Deuxième étape : débordement des crêtes et progression sur les versants. Cette étape coïncide avec la décennie 1960-1970 marquée par un essor économique somme toute remarquable de la ville. Les populations qui ne cessent d'affluer investissent les versants, favorisées en cela par l'amélioration de la voirie. C'est ce qu'on a appelé l'urbanisation « en pétale » parce qu'elle progresse sous forme de lobes à partir des sommets de crêtes et en s'arrêtant vers les bas des versants.

Troisième étape : l'occupation des bas-fonds. Cette dernière étape est relativement récente, car elle date des années 1970. Elle se caractérise par le remplissage systématique des fonds de vallée. En effet, toutes les terres exondées (versants et sommets des collines) étant désormais saturées, les nouveaux migrants qui recherchent la proximité avec le centre ville (zone d'emplois), se lancent dans la conquête des bas-fonds marécageux et les occupent. La boucle se ferme ainsi, traduisant la densification complète des unités morphologiques de la ville.

Les fonds de vallées marécageuses de Yaoundé constituent des sites à risque pour la santé des citadins qui y habitent. Ces risques sont élevés si l'on en juge par le taux de cas de diarrhée $(17,1 \%)$ qu'y a été relevé en $2004^{12}$. Il se trouve que, contrairement aux plateaux et aux versants, les fonds de vallées marécageuses de Yaoundé sont un environnement favorable à l'accumulation des eaux et des détritus pollués. Cette accumulation tient à la fois de la configuration même du site et de la texture des sols en présence. Espaces bas et plats, ces bas-fonds constituent une sorte de réceptacle pour toutes les eaux de ruissellement en provenance des zones situées en hauteur. Ces eaux 
chargées d'immondices s'ajoutent aux rivières qui, du fait de la platitude du site, connaissent un écoulement lent au point de créer par endroits des zones de stagnation. À la moindre averse, l'inondation devient inéluctable du fait de la nullité ou de la faiblesse de la pente, mais plus encore de l'affleurement du gneiss dans le talweg qui ne permet pas aux rivières d'inciser davantage leur lit et, par ricochet, d'augmenter leur capacité de transport. Ainsi s'assemblent à la surface de la roche saine des formations alluviale et colluviale. Révélatrices des mauvaises conditions de drainage, ces formations alluvio-colluvionnaires manquent de véritable cohésion et ont, comme trait morphologique majeur, leur engorgement excessif en eau. Mêlées à du sol, elles forment des sols hydromorphes, localement appelés «elobi». Ainsi, de par leurs caractéristiques pédologique et topographique, les fonds de vallées de Yaoundé sont des espaces qui accumulent des eaux de ruissellement charriant des matières fécales diluées et autres micro-organismes associés aux problèmes de santé qui y sont fréquemment relevés.

\section{Figure 2 - Mécanisme usuel de l'occupation du site urbain à Yaoundé}

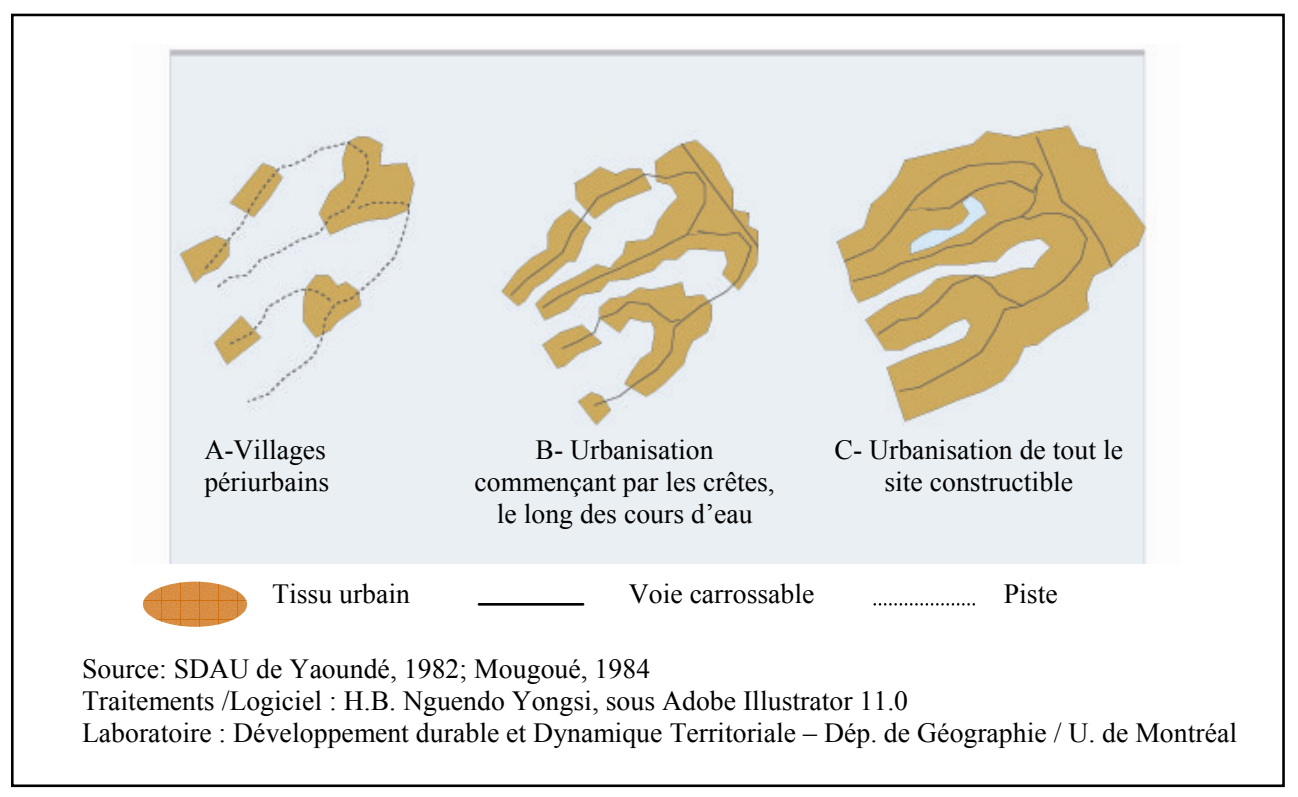

\section{Une mise en valeur différentielle des fonds de vallées de Yaoundé}

L'existence des bas-fonds marécageux sillonnant la ville constitue l'un des traits physiques marquants du site de Yaoundé. Le précédent paragraphe a permis de voir que, malgré les risques qu'ils présentent, ils ne constituent nullement un obstacle à l'urbanisation. Du point de vue de la gestion ou de l'aménagement, si la définition des fonds de vallées se révèle opérationnelle pour les aménageurs et décideurs (zones basses et humides), elle est également précise pour les pouvoirs publics pour qui, juridiquement, les bas-fonds relèvent du domaine public national et sont classés en zones écologiquement fragiles interdites à toute construction. Cette classification a comme conséquence la non-prise en compte des contours de ces bas-fonds dans la délimitation des quartiers administratifs ur- bains et débouche donc sur une difficulté majeure : celle de connaître leur étendue précise. Il revient ainsi à tous ceux qui s'y intéressent de développer une approche méthodologique devant leur permettre d'approcher la réalité. Pour avoir une idée de la surface couverte par ces sous-espaces, nous en avons, dans un premier temps, déterminé les limites précises. Ensuite, nous les avons carroyées $(1 * 1 \mathrm{~cm})$ afin de déterminer leurs superficies réelles sur la base de l'échelle de la carte qui nous a servi de support (carte topographique au 1:15 000). Ce qui nous a permis d'évaluer à près de $40 \%$ l'espace physique de Yaoundé couvert par les bas-fonds.

Les bas-fonds de Yaoundé se répartissent entre les différents bassins versants du site. Ils s'ordonnent autour de la vingtaine de rivières et ruisseaux qui arrosent la ville. Toutefois, suivant l'objet ou les objets 
dominants de leur mise en valeur, nous les avons regroupés en trois catégories (figure 3).

\section{Les bas-fonds aménagés à des fins industrielles}

Très limités dans l'espace urbain, ils correspondent aux fonds de vallées qui supportent des réalisations relevant d'une forme d'urbanisme " pensé ». Car elles sont le fait des pouvoirs publics, qui ont ainsi pris de l'avance sur les populations. Ces installations d'ordre économique sont variées et se rencontrent dans trois fonds de vallées : 1) le fond de la vallée du Ntem, 2) le fond de la vallée marécageuse du Mfoundi et 3) le fond de la vallée de l'Ekozoa.

Le fond de la vallée du Ntem: situé presque au nord de la ville, il est occupé dans son cours moyen par les installations de la Cameroon Rail Corporation. Ces installations comprennent la zone d'embarquement des voyageurs et la zone d'ateliers et d'entreposage des marchandises.

\section{Figure 3 - Typologie des bas-fonds marécageux à Yaoundé}

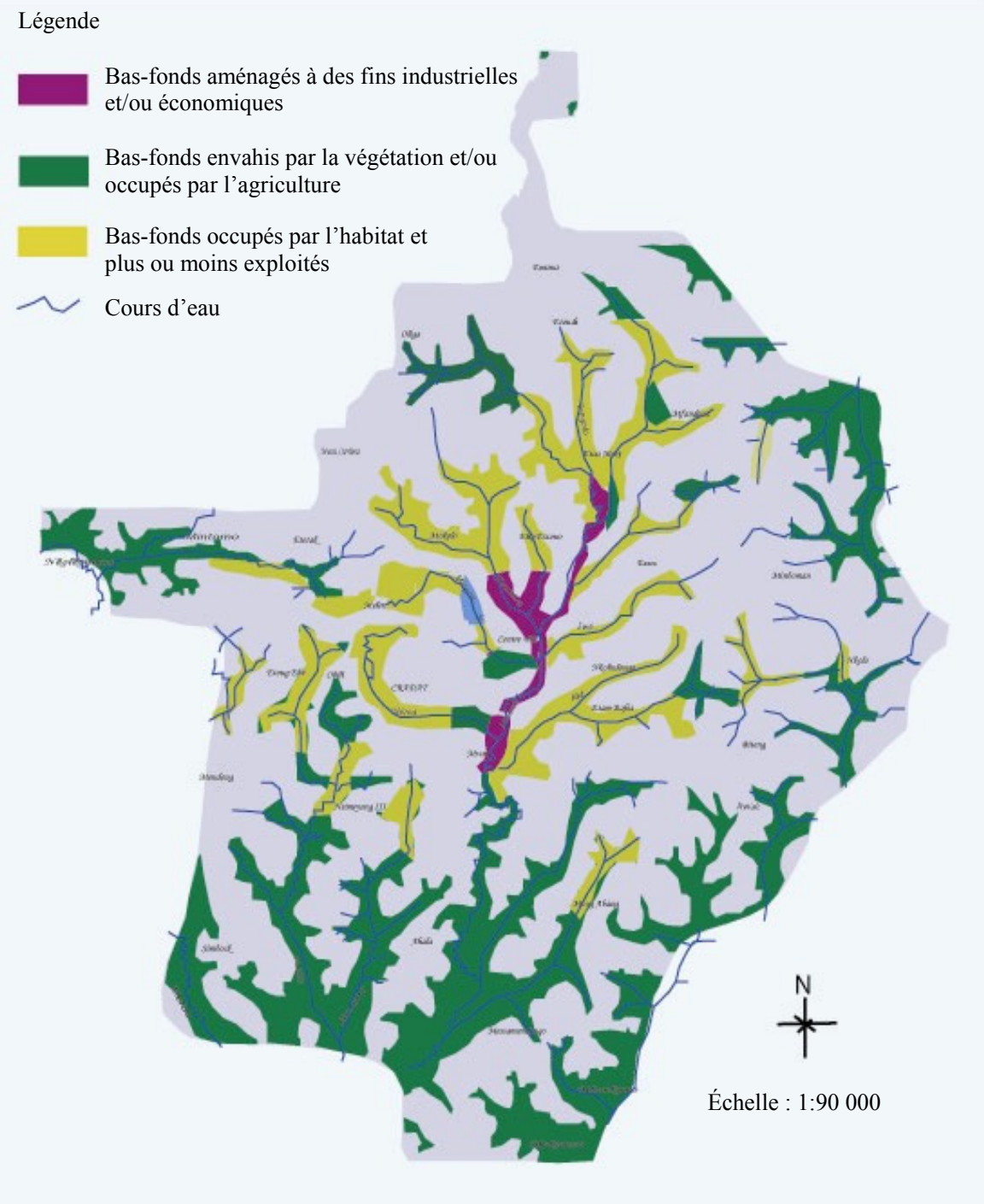

Sources : 1. Fond de carte : CUY/DST/Observatoire urbain

2. Données : Observations de terrain, 2002 et 2004

Traitement et logiciel : H.B. Nguendo Yongsi ; sous Adobe Illustrator 11.0

Laboratoire : Développement durable et Dynamique territoriale - Université de

Montréal 
Le fond de la vallée marécageuse du Mfoundi: le Mfoundi est le principal cours d'eau de la ville. Dans sa section sud, les pouvoirs publics ont aménagé de part et d'autre du lit une zone industrielle qui rassemble les grandes usines de la ville.

Le fond de la vallée de l'Ekozoa: situé au centre ville, il devait recevoir l'aménagement d'un centre tertiaire. Mais actuellement, c'est un espace vert plus ou moins protégé avec des plants d'eucalyptus.

Le moins que l'on puisse dire est que, dans l'ensemble de ces fonds de vallées, les constructions à caractère résidentiel sont inexistantes ou du moins très rares dans un couloir de 20 à 30 mètres. Les pouvoirs publics, en intervenant sporadiquement par des démolitions de maisons, y empêchent ou freinent l'avancée des habitations

\section{Les bas-fonds envahis par la végétation et/ou aménagés à des fins agricoles}

Ils sont assez nombreux (une dizaine) et se localisent surtout dans les périphéries urbaines, où les autochto- nes tentent farouchement de s'opposer à l'afflux d'une population allogène de plus en plus nombreuse, dans laquelle elle voit, depuis une décennie, des envahisseurs.

Parmi ces fonds de vallées encore non anthropisés, on peut citer celui de Nkolkoumou (Nkolbisson) à l'est, et celui de la Mefou au sud-ouest (vers simbock). Très larges et en auge (forme de U), et du fait qu'ils correspondent en général aux points de convergence des ruisseaux, ces bas-fonds sont, pour les populations environnantes, des milieux réservés à la chasse, à la pêche et à la cueillette d'écorces et plantes dont les vertus thérapeutiques sont avérées par le savoir traditionnel local (photographie 1).

Les autres bas-fonds marécageux de la ville ayant une couverture végétale sont ceux mis en culture. Ce qui ne surprend pas quand on sait que les villes africaines sont en général caractérisées par l'existence d'une autoproduction vivrière et d'une agriculture marâ̂chère qui se développent dans les parcelles ou dans les espaces publics laissés vacants.

\section{Photographie 1 - Un bas fond agricole au nord-ouest de la ville (entre le palais des Congrès et le Mont Fébé)}

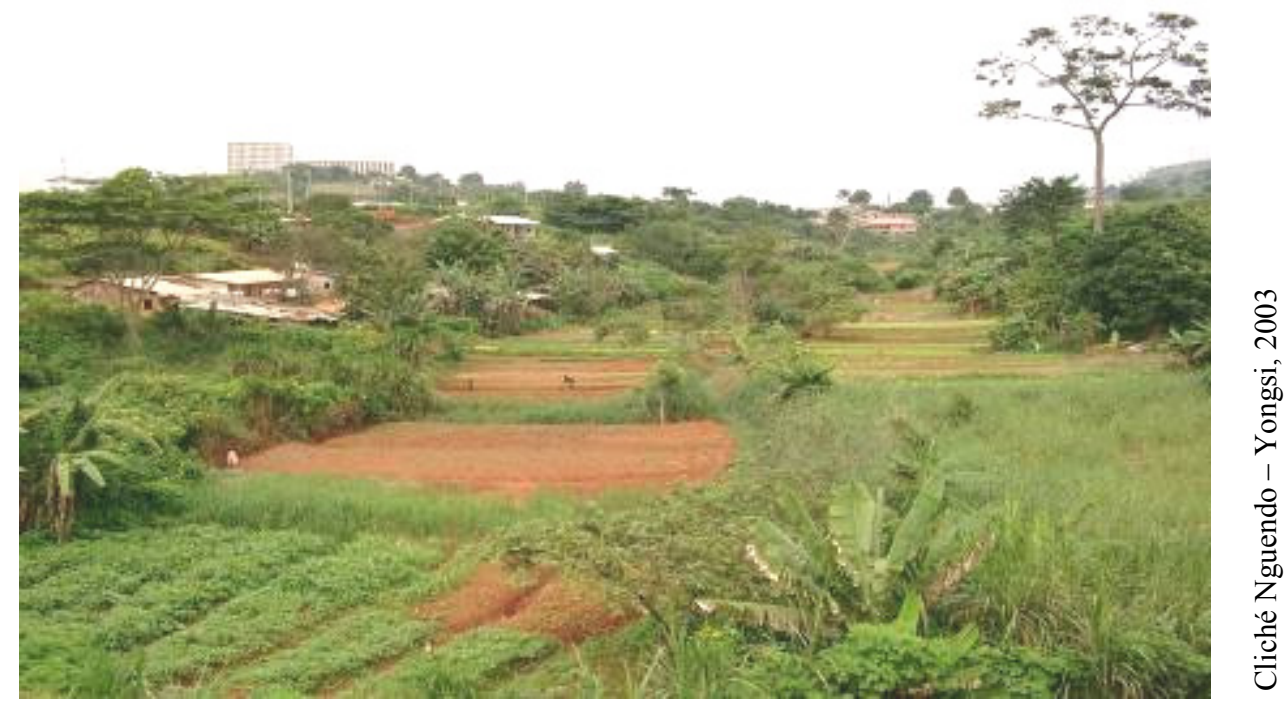

Au premier plan à gauche, des billons de feuilles et légumes; et en arrière plan, des billons et autres casiers en cours de construction pour la mise en terre des céréales

\section{Les bas-fonds occupés par l'habitat et plus ou moins intensément exploités}

Théoriquement, les bas-fonds marécageux de Yaoundé appartiennent aux zones non aedificandi, car indé- pendamment de la législation régissant les constructions dans la ville, ils présentent des caractéristiques physiques peu propices à l'installation humaine. Certains quartiers de la ville s'y sont néanmoins développés. Nombreux sont les bas-fonds urbains qui sont 
ainsi bâtis. La figure 3 montre leur localisation spatiale et leur emprise. L'espace physique est ici dominé par les habitations, certes, mais en outre certaines activités s'y déroulent : la petite agriculture, l'élevage de case, l'artisanat de production, etc. Ceci illustre bien la diversité des situations dans ce type de basfonds. Nous avons parcouru l'ensemble de ces bas-fonds habités et en avons étudié minutieusement un certain nombre. En fonction de la présence (ou non) d'une ou de deux activités dominantes intégrées à l'habitat, nous avons relevé trois types de situation, à savoir : 1) les bas fonds presque exclusivement occupés par l'habitat; 2) les bas fonds occupés par l'habitat, et parsemés de nombreux champs de case et d'enclos d'élevage; 3) les bas-fonds occupés par l'habitat avec des activités artisanales prépondérantes.

Les bas fonds presque exclusivement occupés par l'habitat. Ils sont très peu nombreux. Parmi eux, on a le bas-fond de Nylon-Bastos. Situé à l'ouest du lieu dit rond-point Nlongkak, il fait partie de l'espace administrativement connu sous le nom de Nlongkak 1b. Drainé par deux ruisseaux qui coulent vers la rivière Ekozoa, c'est un bas-fond en « V » qui couvre une superficie d'environ 46 hectares (ha). Coincé entre les versants de Bastos à l'ouest et de Nlongkak à l'est, il n'est desservi que par quelques chemins en terre battue, tracés par les populations et par les eaux de ruissellement. Le paysage de ce bas-fond est donc marqué entièrement par les constructions, dont la plu- part datent des années 1970. C'est un habitat spontané et dense qui ne dispose d'aucun équipement socio urbain.

Les bas fonds occupés par l'habitat et parsemés de nombreux champs de case et d'enclos d'élevage. Nous en avons rencontré plusieurs. Mais le cas le plus illustratif est celui de Obili-Etoug Ebé (photographie 2). Administrativement, il s'insère dans le très ancien quartier populaire d'Obili, dont le nom est issu de la déformation locale du terme français « obligatoire » et qui signale un événement politique du temps colonial français. C'est un bas-fond très peuplé qui rassemble une population composite, faite majoritairement d'allogènes. Le secteur est drainé par la rivière Biyeme qui reçoit ici des filaments de ruisseaux. Les voies secondaires en terre qui partent des versants s'arrêtent brutalement au bas de ceux-ci, mais sont prolongées, dans le talweg entièrement anthropisé, par des pistes constamment boueuses.

Les populations qui s'y sont installées pratiquent deux activités : une petite agriculture et un élevage extensif. La première se fait dans les nombreux interstices qu'on y trouve. C'est donc une agriculture de case, qui porte essentiellement sur les condiments de cuisine (piment, ail, gingembre, tomate), les feuilles et le maïs. Quant à l'élevage, il est composé de volaille (en laisse) et de petit bétail, notamment le porc et la chèvre, à la fois en laisse et en étable.

\section{Photographie 2 - Coexistence habitations-petit élevage, habitations-champ agricole à Etoug Ebé}

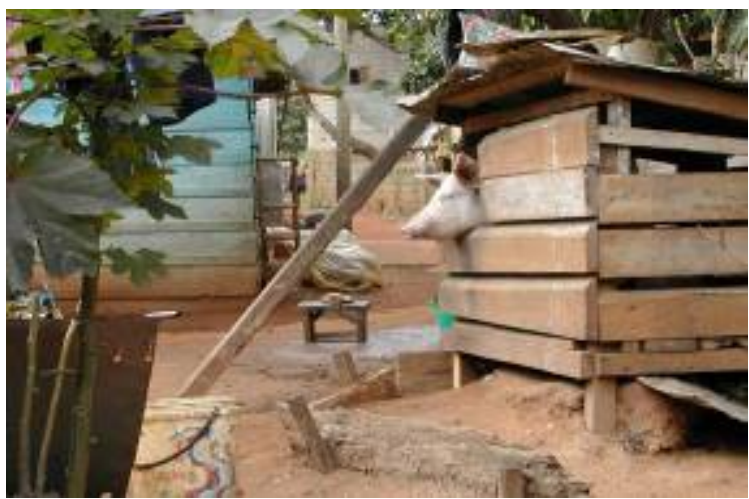

Les bas-fonds occupés par l'habitat avec des activités artisanales prépondérantes: le cas de MokoloBriqueterie. Légèrement incurvé, ce bas-fond est ceinturé des interfluves qui portent les quartiers de Nkomkana au nord, de Messa au sud, de Madagascar
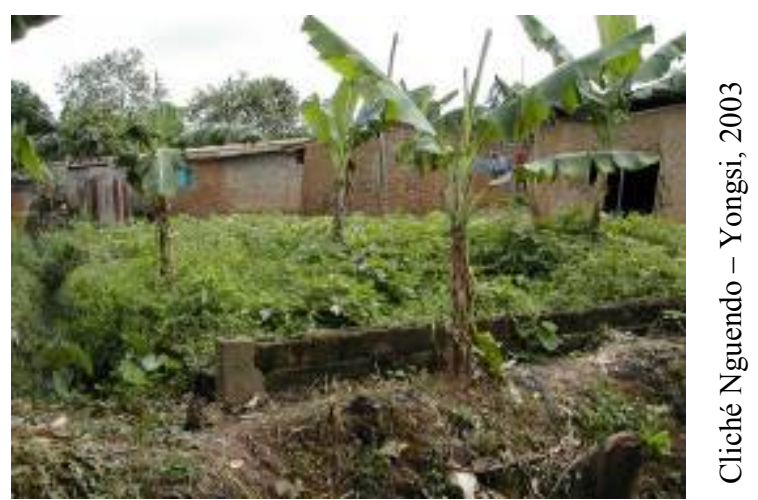

à l'ouest et de Tsinga à l'est. Le bas-fond en lui même est un ensemble spatial homogène du point de vue du bâti, c'est-à-dire très pauvre et non structuré. Il est drainé par la rivière Abiergue, affluent du Mfoundi et par quelques ruisseaux. Deux quartiers contigus re- 
couvrent ce bas-fond : ce sont Mokolo et Briqueterie qui s'étendent sur 121 ha et qui abritaient en 1993 une population estimée à 41000 habitants. Ce qui frappe dans ces quartiers, c'est, d'une part, le désordre dans l'implantation du bâti, la pauvreté de l'habitat et la quasi-absence d'espace libre entre les cases et, d'autre part, la prépondérance d'activités artisanales, aussi polluantes les unes que les autres. Ainsi en est-il des ateliers de vidange de voiture, des structures de fabrication de glace et de sucettes, des garages de réparation automobile, des fonderies, des abattoirs sauvages, etc.

Figure 4 - Des exemples de bas-fonds marécageux occupés par l'habitat et autres activités
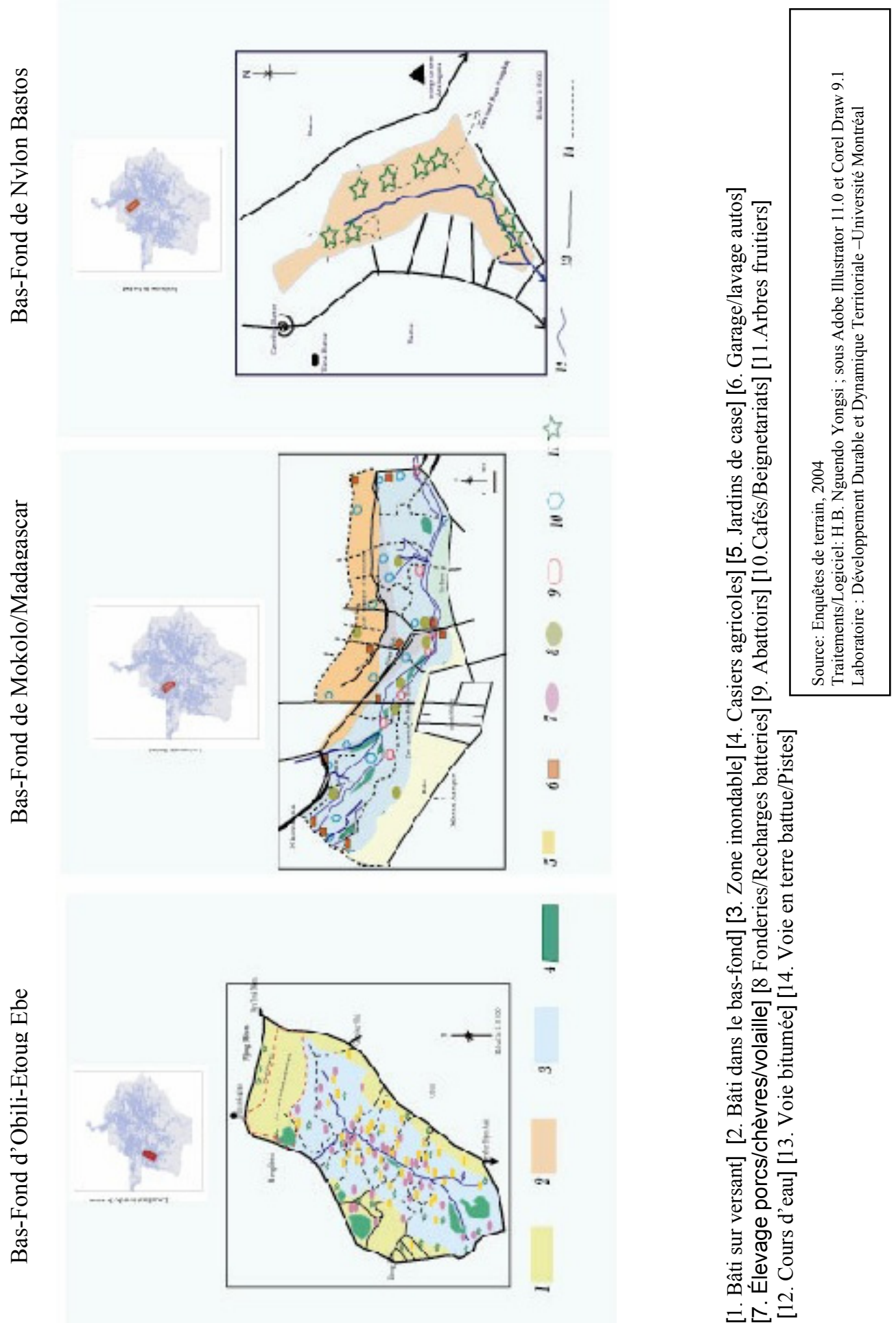


\section{Conclusion}

Au cours de ces vingt dernières années, l'espace physique de la capitale a été à maints endroits surexploité par l'homme. Cette surexploitation se manifeste à travers une série d'artéfacts qui témoignent tous de la capacité des citadins à agir sur l'interface urbaine, de la transformer et d'en user selon ses intentions et ses besoins. À Yaoundé, ce phénomène se voit nettement dans les nombreux fonds de vallées qui dessinent le paysage urbain. Derniers espaces d'anthropisation si l'on s'en tient au mécanisme usuel de l'occupation du site de la ville, ces fonds de vallées sont aujourd'hui plus ou moins occupés et ce, à des fins diverses. Bien que servant d'espace de prédilection pour une agricul- ture qui contribue au ravitaillement de la ville, les basfonds marécageux restent un espace à risques majeurs pour la santé des résidents. Aussi, l'installation des citadins se doit d'y être rigoureusement contrôlée.

\section{Notes et références}

1 United Nations (1995), World Urbanization Prospects: The 1994 Revision, New York, ONU, 178 p.

2 www.unhabitat.org.

3 Mainet, G. et H. Mainet-Valleix (2004), « Les villes africaines: croissance et diversité régionale », dans F. Bart (dir.), L'Afrique, continent pluriel, Liège, SEDES/CNED, p. 177-197. 


\section{Publicité}

\section{Revue internationale PME}

